\title{
De Culioli à Saussure, aller-retour
}

\author{
Dominique Ducard \\ Université Paris-Est Créteil, Céditec EA 3119, Créteil, France
}

\begin{abstract}
Résumé
La linguistique de l'énonciation développée par Antoine Culioli est une théorie des opérations prédicatives et énonciatives. Les deux concepts d'opération et de représentation sont ainsi au cœur du modèle épistémologique et de la méthode d'analyse, qui cherche à démêler les relations en jeu dans la construction des énoncés pour les rapporter à l'activité symbolique de représentation et au processus mental qu'elle présuppose. Un examen critique du modèle des trois niveaux de représentation nous amène à distinguer la fonction de représenter du mode de représenter. Le retour à Saussure nous permet de revoir le modèle en y intégrant, au niveau des représentations notionnelles, ce que celui-ci nomme des figures. Une brève étude de cas illustre, pour finir, le développement théorique.
\end{abstract}

Mots-clés

énonciation, représentation, opération, figure, Culioli, Saussure

\begin{abstract}
The linguistics of enunciation formulated by Antoine Culioli is a theory of predicative and enunciative operations. Thus, the concepts of operation and representation are central to the epistemological model and the analysis method which aims to disentangle the links in play in the construction of utterances to relate them to the symbolic activity of representation and to the mental process that it presupposes. A critical examination of the model of the three levels of representation leads us to distinguish the function of representing from the mode of representing. The return to Saussure enables us to re-examine the model by integrating, at the level of notional representations, what Saussure names figures. In conclusion, a brief case study exemplifies the theoretical development.
\end{abstract}

\section{Keywords}

enunciation, representation, operation, figure, Culioli, Saussure

Les notions de représentation et d'opération sont des notions fondamentales dans la théorie de l'énonciation élaborée par Antoine Culioli. Pour les problématiser nous baserons notre propos sur les entretiens réalisés avec celui-ci, l'entretien étant un genre qui amène le questionné à répondre de ses choix et à s'expliquer sur les concepts. Nous nous focaliserons plus particulièrement sur les moments où il est question de Saussure, en nous intéressant notamment à un entretien avec Simon Bouquet publié dans le numéro de L'Herne, que celui-ci a dirigé, sur Saussure (Bouquet 2003, p. 137-149), au cours duquel Culioli dit avoir réévalué la pensée saussurienne suite à la lecture des textes publiés sous le titre Écrits de linguistique générale (2002).

À la question «Comment vous situez-vous dans les courants de la linguistique contemporaine?», posée lors d'un entretien de 1992 (Lopez Alonso et Séré de Olmos 1992, p.25-57), Culioli répond en posant d'abord qu'il convient de se 
positionner par rapport à des programmes de travail, soutenus par des points de vue théoriques, et non pas par rapport à des écoles ou des courants. Cherchant à traiter de la complexité du langage, sans séparer les problèmes selon des domaines d'étude (lexique, morphologie, syntaxe, sémantique, pragmatique), il dit alors ne pas pouvoir se satisfaire des approches de la grammaire générative ou de la grammaire relationnelle. Et il mentionne les linguistes qui sont convoqués dans ce dossier. À propos de Guillaume il cite une remarque que J. Stefanini lui a souvent faite: «Tu fais ce que Guillaume entrevoyait de faire et qu'il n'a pas pu réaliser parce qu'il vivait à une autre époque» (Lopez Alonso et Séré de Olmos 1992, p.28), il reconnaît avec celui-ci une proximité d'objectif et se demande si Guillaume n'a pas eu une certaine influence sur la façon dont Benveniste a conçu les problèmes de linguistique générale. Il rejoint par ailleurs Bally dans la prise en compte, dans la démarche, de l'activité mentale. Il mentionne à nouveau Benveniste suite à une seconde question sur les textes et personnalités sur lesquels il s'appuierait. De formation philologique et linguistique, il signale avoir suivi certains cours de Benveniste et les cours de F. Mossé, en philologie germanique. Et il déclare : «J'ai eu le sentiment, dans certains cas, que, de façon indépendante, j’ai abouti à des formulations que j'ai redécouvertes chez Émile Benveniste sans savoir si mon indépendance n'était pas illusoire, si mes réflexions n'étaient pas dues aux cours suivis. » (ibid. p. 30). Mais sa reconnaissance de dette s'adresse avant tout à des mathématiciens, des logiciens et psychologues (Grize, Gréco, Bresson, notamment), qu'il a rencontrés ou côtoyés, aussi à des lectures en philosophie, en philosophie du langage, en philosophie des sciences. ${ }^{1}$

C'est parce que j'ai voulu travailler sur les représentations dans le domaine de la linguistique, que j'ai été amené à m'intéresser à l'anthropologie, à la logique, à la psychologie cognitive..., à toute une culture complémentaire qui me manquait. (Lopez Alonso et Séré de Olmos 1992, p. 30)

Ces propos rapportés permettent de marquer le rapprochement entre les linguistes cités et il conviendrait, dans une étude approfondie, de dépasser leur caractère apparemment anecdotique pour montrer en quoi les théories convergent ou divergent, Culioli insistant sur l'«assise épistémologique» de la démarche du linguiste.

\section{LE MODĖLE DES TROIS NIVEAUX DE REPRÉSENTATION}

Nous commencerons par rappeler sommairement le modèle des trois niveaux de représentation, qui permet de poser les concepts majeurs de la théorie et leur interdépendance, notamment ceux que Culioli considère comme les «termes 
indispensables »: d'un côté repérage (il lui avait été proposé de nommer la théorie «théorie des repérages ») et opération, de l'autre représentation.

Ce qui entraîne le concept de marqueur, en tant que représentation matérielle, qui est la trace d'opérations portant sur des représentations d'ordre notionnel. (Lopez Alonso et Séré de Olmos 1992, p. 41)

Cet énoncé peut être complété par celui-ci :

La théorie des trois niveaux de représentation permet de construire des procédures explicites de simulation des opérations cognitives à l'œuvre dans l'activité de langage. (ibid., p. 44)

Et l'activité de langage est appréhendée comme une activité symbolique de représentation. Ces quelques citations suffisent déjà à percevoir le caractère problématique de la notion de représentation.

Il y a donc un niveau 1 , celui des représentations mentales ou cognitives, niveau non accessible directement, au sens de non perceptible; un niveau 2, celui des représentations d'ordre linguistique, des textes produits lors de l'activité énonciative: activité de production et de reconnaissance interprétative de formes interprétables; et un niveau 3, qui est le niveau des représentations métalinguistiques, de la théorisation. Le postulat est que les termes linguistiques sont les marqueurs ${ }^{2}$ des représentations du premier niveau, en tant que représentants matériels, substituts manipulables, et qu'ils portent la trace des opérations non conscientes dans l'activité de langage et dont il faut construire des analogues grâce à un système de représentation métalinguistique.

La mise en correspondance entre les niveaux fait apparaître le double sens du terme de représentation: les formes signifiantes sont des représentants de représentations et le linguiste, par son activité de conceptualisation et de formalisation, cherche à représenter le fonctionnement de ces représentants dans leur relation aux représentations.

On peut dire : il faut métareprésenter grâce à des méta-opérations les opérations dont ces marqueurs sont les représentants - ce qui va me permettre de passer de 2 à 1. (Culioli 1985, p. 17)

Il convient de préciser que le linguiste ne peut rien dire d'autre des représentations mentales que ce qu'il peut en dire de son point de vue et dans

2 Sur les notions de marqueur et de trace voir Ducard (2004). On peut encore citer ce passage d'un entretien au cours duquel le rôle du marqueur dans la démarche est souligné : "Alors donc, la notion de marqueur va être utilisée comme point de départ pour cette simulation, qui fait que grâce à une activité métalinguistique, c'est-à-dire de construction d'un langage explicite et autant que possible maîtrisé qui soit dans une certaine condition d'extériorité par rapport à nous, nous allons simuler ce qui se passe au niveau de ces opérations mentales. Et donc nous allons construire un analogue de ces agencements organisés de marqueurs que l'on appelle des énoncés.» (Culioli 2002, p. 184) 
son domaine, qui est celui des langues et des textes (les suites textuelles comme agencements de marqueurs), par sa pratique d'observation, de manipulation et de raisonnement. Dans le système métalinguistique les représentations mentales sont ainsi traitées comme des représentations notionnelles. Le niveau 1 est ainsi une zone frontière et Culioli renvoie par ailleurs à notre expérience du monde (perception, action, imagination) et notre praxis humaine, notamment nos conduites corporelles, ce qui a donné lieu à l'hypothèse du geste mental sousjacent à l'activité de langage ${ }^{3}$ :

[...] il y a aussi un niveau antérieur et qui est hors du domaine de la linguistique et qui est tout le domaine de l'activité cognitive au sens large, i.e. construction de nos perceptions, de nos goûts, nos dégoûts, nos représentations collectives, des objets avec des propriétés culturellement, physiquement, subjectivement déterminées. (Culioli 1985, p. 16)

D'autres études du langage, dans des domaines connexes, en philosophie et en phénoménologie, en psychologie ou en anthropologie, peuvent alors faire écho à ce que le linguiste met au jour. Nous renverrons, pour ce qui concerne la représentation comme image mentale, liée à la perception et à l'action, aux questions qui ont été posées à Culioli (Ducard 2012), en rappelant, entre autres, la référence aux Stoïciens, avec la phantasia, que l'on traduit par « représentation » ou «présentation » ou encore «impression», mais c'est une impression rationnelle, dans sa relation avec le dicible ou l'exprimable (lekton). Dans sa réponse Culioli renvoie aux catégories phanéroscopiques de Peirce (priméité, secondéité, tiercéité), il signale comment le lekton est devenu pour lui la lexis, au sens de contenu propositionnel non asserté, équivalent à ce que les Scolastiques appellent le «complexe significabile» (signifiable par complexe) ou encore «complexe enuntiabile» (l'énonçable). ${ }^{4}$ Et il se réfère aux concepts husserliens (Husserl 1992) de Gegenwärtigung (présentation-représentation) et Vergegenwärtigung («représentification » selon la traduction de Ricœur).

\section{De Culioli à Saussure}

Culioli nous dit s'être rapproché de la pensée de Saussure ou plutôt s'y être retrouvé dans sa (re)lecture des textes pour partie inédits, publiés en 2002. Ceci dit les propos tenus lors d'un entretien antérieur à la publication des documents trouvés en 1996 relativisent l'effet de découverte d'un autre Saussure que l'entretien avec Simon Bouquet (2003) met en avant. À propos de la démarche

3 Sur le geste mental on pourra se reporter à Ducard (2009).

4 Culioli se dit avoir été heureux de trouver à l'article lexis du Vocabulaire technique et critique de la philosophie publié par Lalande (1867-1963) une définition correspondant à sa conception de la lexis, et d'y avoir découvert la référence aux Scolastiques. 
d'observation des variations d'une langue à l'autre, aussi d'un texte à l'autre dans une langue, d'une situation à une autre et d'un locuteur à un autre, et de la recherche d'invariance, par généralisation et au moyen des procédures d'abstraction, il déclare que le problème du rapport variation/invariance ou labilité (plasticité, déformabilité)/stabilité n'est pas résolu de façon satisfaisante par le couple langue/parole :

Pour tout un ensemble de raisons, j'ai été très vite assez peu satisfait, non pas de la façon dont Saussure a essayé, à mon avis, de poser le problème, du moins si l'on regarde tous les textes (notamment tous les textes manuscrits autour du Cours de linguistique générale) mais de la façon dont cela a été figé dans une certaine tradition. (Ducard 2004, p. 12)

L'opposition entre la langue dans son rôle de «régulateur central» et les «évènements singuliers qui vont nous fournir la parole dans des situations particulières » (Ducard 2004, p. 12), ne permet pas de rendre compte des cohérences internes des énoncés, parlés ou écrits, avec les régularités observables dans le jeu des interactions entre les formes signifiantes. Culioli avance alors l'idée d'une «langue parolière» (ibid., p. 13), entre le subjectif et le transindividuel.

Interrogé par S. Bouquet il revient sur le couple langue/parole à laquelle une certaine interprétation de la langue comme «trésor mental», thesaurus, a pu donner lieu en l'assimilant à un stockage. Réinterprétant l'idée de la «passivité sociale» et du caractère statique de la langue selon Saussure, liés à une forme d'inconscience (ou de semi-conscience), il en vient à déclarer : "c'est en fait tout simplement l'activité mentale de langage» (Culioli 2003, p. 138), activité dont nous n'avons pas conscience et qui se traduit par une transmission, par un échange. Nous pouvons nous reporter ici au reproche que Saussure adresse à la grammaire comparée de ne pas avoir envisagé le langage «sous l'aspect d'un phénomène, et donc son caractère d'exercice d'une faculté de l'âme » (Saussure 2002, p. 130). Saussure dit aussi que «La conquête de ces dernières années est d'avoir enfin placé non seulement tout ce qui est le langage et la langue à son vrai foyer exclusivement dans le sujet parlant soit comme être humain soit comme être social. » (ibid., p. 130). Le problème de la conscience rejoint celui de l'individu par rapport au sujet, avec un rapport dialectique entre le sujet de la parole, le « sujet parlant» et le sujet de la «passivité sociale» : pas de parole sans langue et inversement.

L'insistance sur l'échange, sur la reconnaissance interprétative dans la boucle sémiotique, ce qui fait qu'un énoncé est produit en vue d'être reconnu comme étant produit pour être reconnu et interprétable, avec un énonciateur-coénonciateur, que Culioli dit entrevoir chez Saussure, pose aussi la question du circonstanciel (ce qui est échangé, transmis dans une parole à autrui, à propos de quelque chose et en vue 
de quelque chose, dans un moment et un lieu donnés) et du «hors-circonstances », avec ainsi d'un côté des occurrences linguistiques particulières dans des situations singulières, de l'autre l'adaptabilité et la plasticité de la langue et une aptitude à généraliser, à abstraire, c'est-à-dire une intrication de phénomènes hétérogènes, de l'ordre de l'espace-temps et de l'ordre de «l'idéalité », de la représentation.

Non seulement il ne peut pas y avoir dans la langue du sens qui ne soit, si j'ose dire, de la parole en attente, mais en même temps il ne peut pas y avoir de la parole qui ne s'appuie sur du construit en préalable. (Culioli 2003, p. 145)

Cette affirmation vient après avoir donné l'exemple d'un échange entre deux locuteurs comprenant une réplique avec «quand même», dont il livre une brève analyse, avec une valeur que l'on peut qualifier d'agonistique, comparativement à d'autres valeurs dans d'autres contextes et situations. On peut relever, pour prendre un exemple de «quand même» dans la parole de Culioli, le «quand même» de protestation ou réprobation à propos de la dernière phrase du Cours de linguistique générale, que $\mathrm{S}$. Bouquet présente comme apocryphe et contraire à la pensée de Saussure : «la linguistique a pour unique et véritable objet la langue envisagée en elle-même et pour elle-même» (Saussure 1971, p.317). S'élevant contre «l'enfermement du domaine» que cette phrase semble promouvoir, Culioli s'exclame: "c'est quand même formidable de construire une cage comme cellelà ! »(Culioli 2003, p. 148, souligné par nous). Du 'quand même' prêt à l'emploi, dont il a par ailleurs proposé une analyse dans son séminaire oral, il dit « qu'il est là, comme un exprimable parmi d'autres, latent et donc prêt à être activé. » (ibid., p. 148). Le problème est alors celui de la relation établie entre un construit, la forme 'quand même', dont il faut expliquer la composition et les latitudes combinatoires, selon des règles de compatibilité et d'incompatibilité, avec ses valeurs possibles, et une occurrence dans un contexte et une situation qui en déterminent l'interprétation, selon la construction et la reconstruction des co-énonciateurs dans l'échange, ce qui en fait un 'quand même' polémique, ou de protestation, ou de reproche ou de satisfaction, de soulagement, etc. Et de toutes les occurrences de la séquence, fournies par un corpus de textes issus de paroles diverses, il faudrait tirer, dans un système de représentation métalinguistique, une forme abstraite : la forme schématique, qui permettra, par la formalisation - une description ou une notation algébrique ou un graphe - de rendre compte de sa déformabilité et des opérations dont elle est le marqueur. Polyopération puisque nous avons d'une part une opération de construction d'un existant spatio-temporel, avec 'quand', d'autre part une opération d'identification avec 'même', qui, par la coaptation (Benveniste) des deux termes, et en fonction de la suite textuelle, avec son contour prosodique, dans laquelle l'expression apparaît, va faire que l'état de chose situé par 'quand' est 
identifié à l'état de chose qu'il est; état de chose qui est signifié, dans notre exemple, par le syntagme «construire une cage comme celle-là». Dans la prédication nous avons d'un côté la notion de $\left\langle\right.$ cage $>^{5}$ - la représentation dont le représentant lexical est le mot «cage»-et d'un autre côté l'occurrence de la notion par renvoi anaphorique « comme celle-là », avec un « comme» qui est un opérateur de qualité (une cage comme la cage que c'est). À quoi il faut ajouter la valuation de cet état de chose par le prédicat «formidable», qui marque, dans son entourage, surprise et réprobation.

La mise en relation est, selon Culioli, fondamentale et omniprésente chez Saussure; elle définit ce qu'est une opération, toute relation étant en elle-même une abstraction.

Ce qui a changé mon regard sur Saussure, c'est de découvrir qu'il était hanté par ces mises en relation. Or ce sont ces dernières qui construisent la cohérence d'un discours, d'un texte (quand on dit qu'un énoncé fait sens, il fait d'abord texte). Et ce texte, dans une intrication permanente, est formé d'opérations - relevant d'une activité qui est aussi bien activité de représentation que de régulation, car elle est inscrite dans l'action-; ces opérations, qui sont des catégorisations, des schématisations, etc. vont se trouver entrer directement dans un échange qui se produit dans des circonstances et à un moment particuliers, tout le système de ces opérations étant un dispositif qui leur permet, justement, de s'appliquer par référence, par renvoi, à des situations particulières. (Culioli 2003, p. 146)

Trois grandes catégories d'opérations sont dégagées par l'analyse de l'activité de langage: de Représentation, de Référenciation, de Régulation (les trois R). Dans l'énoncé exclamatif mentionné ci-dessus nous retrouvons les trois types d'opérations avec la construction d'une représentation (R1) valuée en référence à un état de chose (R2) et transmise à autrui en vue d'un assentiment (R3). Nous pouvons alors considérer que la représentation est un opérande 6 à partir duquel des opérations, prédicatives et énonciatives, sont effectuées et que cet opérande est redéfini à chaque étape de la construction de l'énoncé. Et nous pourrions adopter la définition qui est donnée de l'opération en logique: détermination de la valeur d'une relation liant plusieurs variables.

5 Nous avons adopté les chevrons quand il s'agit de la notion, les guillemets simples pour la figure (au sens saussurien, voir plus loin), porteuse de toutes ses valeurs possibles en langue et en attente de la signification contractée lors de son emploi, les guillemets français pour les emplois.

6 Un opérande est, en mathématique, une «donnée, quantité ou valeur, entrant dans une opération arithmétique ou logique», et, en informatique, une "valeur utilisée dans un traitement»; c'est aussi, en linguistique transformationnelle, une «phrase, élémentaire ou non, sur laquelle s'applique une transformation » (définitions du TLFi données sur le portail lexical du Centre National de Ressources Textuelles et Lexicales, en ligne: http://www.cnrtl.fr/ definition/operande). Culioli rappelait fréquemment, dans son séminaire, sans plus de précision, qu'il n'y a pas d'opération sans opérande. 
La mise en relation correspond par ailleurs, toujours selon Culioli, à la différence saussurienne, sur laquelle il revient en citant la formule de Saussure parlant « de la diversité des signes dans l'idée une ou de la diversité des signes dans l'idée diverse», tirée d'une note de «L'essence double du langage» (Culioli 2003, p. 141). Il voit dans la diversité des signes selon Saussure, avec le postulat de non synonymie, ce qu'il nomme la déformabilité à travers le multiple et le foisonnement sémantique. Les signes s'altèrent les uns les autres, dans le temps selon Saussure, par "déplacement du rapport total entre signifiant et signifié » (Saussure 2002, p. 329); pour Culioli les notions, organisées selon un rapport intérieur/extérieur, supposent une altérité ; pour l'un comme pour l'autre l'identité est différentielle, la valeur est relative.

Le domaine de la linguistique, plus généralement de la sémiologie, est celui de la «pensée relative» : des idées relativement à des signes, des signes relativement à des idées. Le principe saussurien de la relativité générale trouve son expression la plus forte dans ce que Saussure nomme le «quaternion final» (ibid., p. 42), composé de quatre termes : signe, forme, signification, figure, et de trois rapports : une forme relative à une signification (et inversement), une forme relative à d'autres formes (différence générale des formes n'existant que selon la différence des significations), une signification relative à d'autres significations (différence générale des significations n'existant que selon la différence des formes), triple rapport auquel s'adjoint la figure vocale, qui devient forme signifiante quand elle intègre le rapport sémiologique. Nous verrons plus loin comment ce schéma sémiologique permet de reposer le cadre des trois niveaux de représentations.

Si Culioli reconnaît qu'il y a un air de famille entre ce qu'il pense et fait et ce qu'il lit de Saussure, il convient de manier les ressemblances avec précaution; il n'y a pas plus de synonymie dans des concepts jugés similaires que dans des mots usuels considérés comme sémantiquement équivalents. Culioli, prudent, dit ainsi : «je pense comme lui, ou plutôt comme je pense qu'il pense.»(Culioli 2003, p. 146).

\section{De Saussure À Culioli}

Le terme de représentation, que nous avons revisité au regard de celui d'opération, mérite aussi d'être révisé quand il s'applique aux trois niveaux du modèle. Les rapports fléchés établis entre ces trois niveaux (le niveau 3 renvoie à la relation entre le niveau 2 et le niveau 1) justifient un emploi de représentation qui correspond à la fonction de représenter: le formulaire métalinguistique cherche à représenter les processus cognitifs et affectifs appréhendés à travers les traces que portent les textes générés dans l'activité d'énonciation. Mais dans la mesure où l'activité de langage est de la "pensée relative», le mouvement de la pensée en 
langue, il paraît difficile de conserver la vicariance que suppose ici la fonction de représenter pour les représentants linguistiques que sont les textes. Les formes qui sont analysées comme des marqueurs ne tiennent pas lieu de représentations mentales, elles en sont les capteurs et les déclencheurs. ${ }^{7}$

Le formulaire métalinguistique est par ailleurs un mode de représenter (métalangage naturel, logique formelle, topologie...), tout comme le langage est un mode de représenter et on pourra alors parler éventuellement, en ce sens, de représentation sémantique. Ce mode de représenter est alors équivalent à un mode de signifier (ou signifiance selon Benveniste). C'est le rapport forme-signification de Saussure, rapport délimité à chaque occurrence par différenciation dans un système de rapports multiples, c'est la mise-en-relation de notions et d'occurrences notionnelles et l'assignation de valeurs parmi les valeurs possibles. La démarche d'investigation du sens procède alors par la glose, glose ordinaire dans le cours de l'énonciation -l'épi-métalinguistique -, que le linguiste reprend à son compte, et glose métalinguistique, depuis l'exploration de familles paraphrastiques, le dépliement forcé des plis du sens, à l'aide de manipulations, de reformulations et de raisonnements contrôlés, jusqu'à la notation formelle ou le graphe. Elle doit composer avec d'un côté des notions lexicales, de l'autre des notions grammaticales, avec des catégories de langue et des schémas morphosyntaxiques et prosodiques. De plus il faut travailler avec la variété des types de situation et de discours, des genres textuels et des registres stylistiques. Nous pouvons ici introduire l'équation de Saussure définissant le domaine du signe, dans son intégralité : « Sémiologie $=$ morphologie, grammaire, syntaxe, synonymie, rhétorique, stylistique, lexicologie etc. le tout étant inséparable» (Saussure 2002, p. 45). Toute analyse linguistique est de ce point de vue nécessairement réductrice.

Si nous suivons la ligne sémiologique de Saussure, le signe se forme « sous notre front, et sa nature (matérielle ou immatérielle, peu importe) est COMPLEXE» (ibid., p.131) et il est impossible de concevoir séparément l'idée et le signe (signifiant), seule la figure peut être opposée au complexe forme-signification ${ }^{8}$. Et il précise :

Si l'on veut, tout signe est une opération d'un ordre psychologique simple -c'est pourquoi [il] ne frappe pas -, mais on ne peut pas parler tout le temps de cette opération en face des délimitations. (Saussure 2002, p. 132)

7 Les termes de capteur et de déclencheur sont utilisés par Culioli pour définir ce qu'est un marqueur.

8 «Parmi les choses qui peuvent être opposées au son matériel, nous nions, essentiellement et sans aucune défaillance future dans le détail, qu'il soit possible d'opposer l'idée. Ce qui est opposable au son matériel c'est le groupe son-idée, absolument pas l'idée. » (Saussure 2002, p. 202). 
Ce que nous interprétons ainsi: il y a des opérations non conscientes de formation mentale des signes, à côté de ou sous ou avec les délimitations des rapports formes-significations, par opposition relative.

Ce qui nous conduit à préciser le modèle des trois niveaux, avec un système de représentation métalinguistique qui a pour fonction de représenter, selon un mode de représenter adéquat à ce qu'il vise, les opérations de mise en relation entre le niveau des représentations notionnelles associées à des figures (niveau 1) et le niveau des représentations sémantiques (en incluant la syntaxe, avec la prosodie, et la pragmatique) (niveau 2). Assembler notions et figures permet de penser le soubassement de l'activité énonciative qu'est l'activité épilinguistique en restant dans le domaine entier du langage. L'épilinguistique, non conscient, désignera ainsi cette activité interne qu'est le mouvement de l'imagination qui fraye des chemins par interconnexion entre notions et figures, créateur d'affinités de sens et de différenciation, et qui se traduit dans le jeu des formes et des significations d'une langue. Ce mouvement - qualifié d' «anamorphose permanente» par Culioli (Culioli et Normand 2005, p. 110) - relève de ce qu'il nomme une «rationalité silencieuse», à côté de la «rationalité d'effectuation démonstrative explicite» (ibid., p. 73), celle qui opère, dans un échange discursif, quand on dit quelque chose pour autrui, à propos de et en vue de. Nous pourrions alors rapprocher la «zone de turbulence $\gg{ }^{9}$ où se connectent les éléments de la langue, libre de la régulation qui opère dans la parole échangée et l'activité discursive, de ce que Lacan nomme «lalangue» ou de ce que Kristeva circonscrit avec la «chora sémiotique» (Kristeva 1977), aussi de ce que Saussure évoque avec les « liaisons associatives ».

\section{POUR FINIR: UNE BRÈVE ÉTUDE DE CAS}

Afin d'exemplifier la démarche du linguiste de l'énonciation, qui part des textes pour comprendre les mises en relation que sont les opérations, il nous a paru utile de présenter une étude de cas, restreinte mais significative. Nous partirons de l'explication suivante de Culioli, donnée au cours de l'entretien sur l'épistémologie et la méthode :

Le concept d'opération permet de simuler la construction d'un énoncé : à partir de représentations notionnelles nous allons construire des termes qui vont pouvoir être pris dans une relation prédicative, construire un espace énonciatif (un référentiel) et construire l'insertion de la relation prédicative dans cet espace. Nous avons un état antérieur, un opérateur qui opère sur cet état antérieur de telle manière que nous allons avoir un résultat qui va être le produit de cette opération.» (Lopez Alonso et Séré de Olmos 1992, p. 42) 
Et nous reprendrons, pour le prolonger, un exemple que Culioli tire d'une thèse et mentionne en passant à propos du modèle des trois niveaux :

On dit, par exemple: "Il a agi avec sa cruauté habituelle», on ne peut pas dire pour des raisons sémantiques qui apparaissent clairement: "avec sa cruauté inhabituelle». On va dire «il agit avec une cruauté inhabituelle»; on ne peut pas dire: "il a agi avec la cruauté habituelle», mais on va dire «il a agi avec la cruauté habituelle chez les gens de son genre».

On voit que le et un sont liés à la présence de habituel ou inhabituel, et que l'on retrouve les problèmes de construction de la détermination, de prédication existentielle et d'aspect. Or si on n'a pas ces trois niveaux [de représentation], que va-t-on faire? On va prendre à chaque instant pour un raisonnement métalinguistique ce qui, en fait, n'est qu'une sorte de paraphrase, de glose qu'on fait sur le texte; ou bien, on va utiliser un langage logique, par exemple, mais on ne va pas se préoccuper de l'adéquation entre le niveau 2 et le niveau 1 . La théorie des trois niveaux de représentation permet de construire des procédures explicites de simulation des opérations cognitives à l'œuvre dans l'activité de langage. (ibid., p. 43-44)

La manipulation de l'énoncé initial fait apparaître des observables qui sont autant de problèmes linguistiques dont il faut essayer de rendre compte pour comprendre les règles de compatibilité ou d'incompatibilité. Passons sur la construction verbale à un temps composé, qui relève d'une opération de référentiation situant l'action dans un intervalle adjacent à un repère origine $\mathrm{t}^{\circ}$. Plusieurs relations prédicatives sont intriquées: une relation entre un agent et l'action qui le définit en tant qu'agent: $<$ il $>$ sert de repère à $<$ agir $>$, noté $<$ il $\underline{\varepsilon}$ agir $>^{10}$, et cette première relation est repérée par rapport à $<$ cruauté $>$ (la propriété $<$ être-cruel>), repérage marqué par l'opérateur d'accompagnement (concomitance) 'avec', d'où résulte l'énoncé $i l$ a agi avec cruauté dans un espace référentiel. Comme le repéré peut se ramener à du «localisé» (au sens abstrait) ou à de «l'identifié » par rapport à un terme qui sert de repère, on dira ici que l'action localisée dans l'agent et située dans un espace-temps (une occurrence de) est identifiée à un acte de cruauté, dans sa représentation.

En introduisant la notion $<$ habitude $>$ dans la relation on s'aperçoit que l'on ne peut pas avoir *il a agi avec cruauté habituelle. Le qualificatif, en raison de la valeur aspectuelle de la notion lexicale $<$ habitude $>$, construit une occurrence (fréquente) de <cruauté $>$ qui va à l'encontre de la seule représentation que nous avons dans la séquence avec $\varnothing$ cruauté. La détermination du terme ${ }^{11}$ permet d'avoir l'énoncé il a agi avec sa cruauté habituelle, par renvoi à des occurrences de

10 Le symbole $\underline{\varepsilon}$, mixte du signe d'appartenance, d'inclusion et d'équivalence, note l'opérateur de repérage epsilon. L'écriture $a \underline{\varepsilon} b$ se lit «a sert de repère à $b$ ».

$11 \mathrm{La}$ détermination nominale relève d'un schème d'individuation, la notion étant fragmentée pour qu'il y ait extraction d'une occurrence (un cas de <cruauté $>$ ) et fléchage (une cruauté particulière... la cruauté de...). 
la notion localisées dans l'agent, à qui est attribué la propriété <être-cruel>, ou encore l'énoncé proposé par Culioli : il a agi avec la cruauté habituelle chez les gens de son genre, avec un agrégat d'individus (le terme agrégat est parfois préféré à celui de classe, trop dépendant d'une logique des classes, pour désigner tous les représentants des occurrences abstraites d'une notion), dont l'agent est un exemplaire, un cas à la fois quelconque et non quelconque (un représentant parmi d'autres, équivalents), et qui sert de repère de localisation. L'opération de fléchage est marquée par le déterminant défini (la cruauté de). L'énoncé il a agi avec la cruauté habituelle est quant à lui suspendu à l'attente d'une localisation compatible avec la valeur itérative du procès ${ }^{12}$. Alors que la construction d'une occurrence, au caractère unique, avec le déterminant indéfini, est incompatible avec la mise en équivalence des occurrences des actions de la classe: *il a agi avec une cruauté habituelle. Ce qui est aussi lié au caractère dense, non discrétisable, de la notion $<$ cruauté $>$. On pourrait avoir, avec une propriété différentielle permettant de discrétiser la notion: il a agi avec une cruauté d'hyène.

Le complémentaire de la notion que représente le prédicat négatif 'inhabituel (le)', en tant que marqueur d'altérité (sortie de la zone intérieure du domaine notionnel $<$ habitude $>$ ), est compatible avec la construction de l'existence d'une occurrence particulière, distinguée et contraire à l'action de l'agent conçue de façon générique par sa réitération: il a agi avec une cruauté inhabituelle, que l'on peut gloser par: il a agi avec cruauté, ce qui n'est pas conforme à sa façon d'agir habituelle. Mais étant donné que la notion de $<$ cruauté $>$ est graduable, nous pouvons comprendre cet énoncé comme signifiant qu'il a agi de façon cruelle mais pas au degré habituel de cruauté. Et il faudrait alors expliquer pourquoi nous interprétons ce degré comme un degré plus fort que celui attendu, qui est préconstruit par le prédicat 'inhabituel(le)' appliqué à 'cruauté', cette orientation en plus ou en moins variant par ailleurs selon les prédicats (il conduit à une vitesse inhabituelle peut être interprété en conduire plus vite ou conduire moins vite qu'à son habitude, selon le contexte).

Nous sommes alors dans la zone frontière des représentations notionnelles, «faisceaux de propriétés d'ordre physico-culturel», un mixte de subjectif et de transindividuel, d'imaginaire et de symbolique, dépendant de l'expérience corporelle du monde, aussi de normes et de croyances. Le plaisir à faire souffrir autrui, physiquement ou moralement, ou l'indifférence à la souffrance d'autrui, par manque de compassion, est l'objet d'une valuation différente selon les individus et les sociétés. Il a agi avec la cruauté habituelle est énonçable si l'on spécifie une

12 La glose du dictionnaire définit ainsi une habitude comme une « façon permanente, fréquente, régulière ou attendue, d'agir, de sentir ou de se comporter, acquise volontairement ou non ", et paraphrase l'expression «avoir des habitudes » par «avoir une vie réglée par des actes qui se répètent». (TLFi, en ligne: http://www.cnrtl.fr/definition/habitude). 
classe de comportements (en ce genre de situation, dans ce cas de figure) où agir ainsi est une norme dans certaines circonstances. Outre les représentations notionnelles, telles qu'elles sont signifiables et exprimables dans les catégories linguistiques, et les opérations sur ces représentations pour construire des énoncés, nous avons à nous interroger sur une catégorie plus générale, qui est ici celle du continu, fondée sur un schéma d'identification d'états de choses les uns avec les autres, dans leur succession, en faisant abstraction des différences. Action sur le monde, itération, généricité, continuité, nous ouvrent alors des vues sur le schématisme de la pensée.

\section{BIBLIOGRAPHIE}

Culioli, Antoine, 1985. Notes du séminaire de DEA 1983-1984, Université de Paris 7, département de Recherches linguistiques, Poitiers.

- 2002. Variations sur la linguistique. Entretiens avec Frédéric Fau, Paris, Klincsieck.

- 2003. «Un linguiste face aux textes saussuriens», Bouquet, Simon (dir.), Saussure, Editions de L'Herne, 2003, 137-149.

Culioli, Antoine et Normand, Claudine, 2005. Onze rencontres sur le langage et les langues, Paris, Ophrys.

Culioli, Antoine et Ducard, Dominique, 2012. «Un témoin étonné du langage », Normand, Claudine et Sofia, Estanislao (dir.), Espaces théoriques du langage, Louvain-la-Neuve, academia, 129-172.

Ducard, Dominique, 2004. «De l'énonciation à la "grammaire subjective". Entretien avec Antoine Culioli (2000)», Entre grammaire et sens, Ophrys, 7-20.

- 2009. «Le graphe du geste mental dans la théorie énonciative d'A. Culioli», Cahiers Parisiens / Parisian Notebooks, volume 5, Les linguistes et leurs graphiques, The University of Chicago Center in Paris, 555-576.

Husserl, Edmund, 1992 [1931]. Méditations cartésiennes. Introduction à la phénoménologie, traduction par Peiffer Gabrielle et Lévinas Emmanuel, Paris, Vrin.

Kristeva, Julia, 1977. «Le sujet en procès », Polylogue, Paris, Seuil, 55-106.

Lopez Alonso, Covadonga et Séré de Olmos, Arlette, 1992. Où en est la linguistique? Entretiens avec des linguistes, Paris, Didier Érudition.

Saussure, Ferdinand de, 1971 [1916]. Cours de linguistique générale, Bally, Charles et Sechehaye, Albert, Paris, Payot.

- 2002. Écrits de linguistique générale, Bouquet, Simon et Engler, Rudolf (éd.), Paris, Gallimard. 